PROCEEDINGS OF THE

AMERICAN MATHEMATICAL SOCIETY

Volume 125, Number 11, November 1997, Pages 3347-3354

S 0002-9939(97)03961-0

\title{
ON THE EIGENVALUES OF THE $p$-LAPLACIAN WITH VARYING $p$
}

\author{
YIN XI HUANG
}

(Communicated by Palle E. T. Jorgensen)

Abstract. We study the nonlinear eigenvalue problem

$$
-\operatorname{div}\left(|\nabla u|^{p-2} \nabla u\right)=\lambda|u|^{p-2} u \quad \text { in } \Omega, \quad u=0 \quad \text { on } \partial \Omega,
$$

where $p \in(1, \infty), \Omega$ is a bounded smooth domain in $\boldsymbol{R}^{N}$. We prove that the first and the second variational eigenvalues of (1) are continuous functions of $p$. Moreover, we obtain the asymptotic behavior of the first eigenvalue as $p \rightarrow 1$ and $p \rightarrow \infty$.

\section{INTRODUCTION}

In this paper we study the nonlinear eigenvalue problem

$$
-\Delta_{p} u=\lambda|u|^{p-2} u \quad \text { in } \Omega, \quad u=0 \quad \text { on } \partial \Omega,
$$

where $p \in(1, \infty), \Omega$ is a bounded smooth domain in $\boldsymbol{R}^{N}$, and $\Delta_{p}=\operatorname{div}\left(|\nabla u|^{p-2} \nabla u\right)$ is the $p$-Laplacian. We are mainly interested in the continuity of the eigenvalue $\lambda$ as a function of the parameter $p$ and its asymptotic behavior as $p \rightarrow 1$ and $p \rightarrow \infty$.

The $p$-Laplacian has attracted growing interest, and figures in a variety of applications in applied fields. For example, it appears in non-Newtonian fluids $(p>2$ for dilatant fluids and $1<p<2$ for pseudoplastic fluids), in reaction-diffusion problems, in flow through porous media, in nonlinear elasticity, in petroleum extraction $([\mathrm{D}])$, and in torsional creep problems $([\mathrm{K} 1])$.

Our study is mainly motivated by the "jumping nonlinearity" problem

$$
-\Delta_{p} u=f(x, u)
$$

with $f(x, u)$ satisfying conditions of the following type:

$$
\lambda_{k}(p) \leq \liminf _{t \rightarrow \rho_{1}} \frac{f(x, t)}{|t|^{p-2} t} \leq \limsup _{t \rightarrow \rho_{2}} \frac{f(x, t)}{|t|^{p-2} t} \leq \lambda_{k+l}(p),
$$

where $\rho_{1}, \rho_{2}=0^{+}$or $\pm \infty$, and $\lambda_{k}(p)$ is the $k$-th eigenvalue of $(0.1)$. In the case $p=2$, sophisticated techniques have been developed and deep results have been obtained by many mathematicians in the past decades. See [LM] for more details and references. However, for $p \neq 2$, those well established and widely employed tools are no longer applicable due to the nonlinear and nonsymmetric nature of the

Received by the editors June 14, 1996.

1991 Mathematics Subject Classification. Primary 35P30, 35 B30.

Key words and phrases. Eigenvalues, the $p$-Laplacian.

Research is partly supported by a University of Memphis Faculty Research Grant.

(C)1997 American Mathematical Society 
$p$-Laplacian. One naturally speculates that, via certain continuation arguments, one may be able to derive some properties of the $p$-Laplacian from the special case $p=2$. In doing so certain homotopy type results will be crucial. As a matter of fact, a similar procedure (in essence) was used in the O.D.E. $(N=1)$ case to study the existence of solutions to a problem of the type (0.2) by Del Pino, Elgueta and Manasevich ([DEM]), where the continuity of $\lambda_{k}(p)$ as a function of $p$ is obvious. Meanwhile, Lindqvist carried out a series of studies in this direction: the dependence on $p$ for the solutions of $-\Delta_{p} u=f(x)$ in [L1], and the continuity of $\lambda_{1}(p)$ and $u_{1}(p)$ (the first eigenfunction associated with $\left.\lambda_{1}(p)\right)$ in [L4]. Recently Kawohl [K1] discussed the behavior of solutions to the problem $-\Delta_{p} u=1$ as $p \rightarrow 1$ and $p \rightarrow \infty$, which appears in torsional creep problems.

In this paper we obtain the continuity of $\lambda_{k}(p)$ for $k=1,2$, thus extend the results in [L4], and derive partial results regarding the asymptotic behavior of $\lambda_{1}(p)$ as $p \rightarrow 1$ and $p \rightarrow \infty$. We note that even though our continuity result is expected to be true by most experts and thus is not surprising at all, its proof is not that trivial. Moreover, our proof reveals an interesting fact, that is, the continuity of the eigenvalues on $p$ follows from the linear independence of the eigenfunctions (see Remark 2.2). The latter is not yet proved except for the O.D.E. case (i.e. $N=1$ ) when $p \neq 2$.

We organize this paper as follows. In Section 1 we introduce notations needed in the sequel. In Section 2 we prove the continuity of $\lambda_{k}(p)$ by constructing appropriate sets of genus $k$ for $k=1,2$. Finally we discuss in Section 3 the asymptotic behavior of $\lambda_{1}(p)$.

\section{Notation}

Consider the eigenvalue problem

$$
-\Delta_{p} u=\lambda|u|^{p-2} u \quad \text { in } \Omega, \quad u=0 \quad \text { on } \partial \Omega,
$$

where $p \in(1, \infty), \Delta_{p} u=\operatorname{div}\left(|\nabla u|^{p-2} \nabla u\right)$ is the $p$-Laplacian, and $\Omega$ is a bounded smooth domain in $\boldsymbol{R}^{N} . u \in W_{0}^{1, p}(\Omega)$ is a solution of (1.1) if for some $\lambda \in \boldsymbol{R}$ and any $\varphi \in C_{0}^{\infty}(\Omega)$,

$$
\int_{\Omega}|\nabla u|^{p-2} \nabla u \nabla \varphi=\lambda \int_{\Omega}|u|^{p-2} u \varphi .
$$

In this paper, all integrals will be on $\Omega$ unless otherwise stated.

We introduce notation we need later in this paper. Let

$$
U_{p}=\left\{u \in W_{0}^{1, p}(\Omega):\|u\|_{p}=1\right\},
$$

where $\|\cdot\|_{p}$ is the norm in the $L^{p}$ space, and

$$
\Gamma_{k}(p)=\left\{A \subset U_{p}: A \text { is symmetric, compact, } \gamma(A)=k\right\},
$$

where $\gamma(A)$ is the genus of $A$, i.e. the smallest integer $k$ such that there exists an odd continuous map from $A$ to $\boldsymbol{R}^{k} \backslash\{0\}$. See e.g. [R] and [Sz] for details of the genus. By the standard Ljusternik-Schnirelmann theory, see e.g. [AA] and [Sz], and the known regularity theory, $\mathrm{cf}$. $[\mathrm{Eg}]$ and $[\mathrm{T}]$, we have the following

Proposition 1.1. There exist $u(k, p) \in A(k, p) \in \Gamma_{k}(p)$ and $\lambda_{k}(p)$ such that

$$
\lambda_{k}(p)=\int|\nabla u(k, p)|^{p}=\sup _{u \in A(k, p)} \int|\nabla u|^{p}=\inf _{A \in \Gamma_{k}(p)} \sup _{u \in A} \int|\nabla u|^{p},
$$


and

$$
-\Delta_{p} u(k, p)=\lambda_{k}(p)|u(k, p)|^{p-2} u(k, p) .
$$

Moreover, for some $\alpha(k, p)>0$ and $\beta(k, p)>0$,

$$
|\nabla u(k, p)(x)-\nabla u(k, p)(y)| \leq \beta(k, p)|x-y|^{\alpha(k, p)},
$$

for $x, y \in \bar{\Omega}$.

Without loss of generality, we assume $|\Omega|=1$. In fact, if $|\Omega|=a$, we define

$$
\Omega^{\prime}=\left\{y: a^{1 / N} y \in \Omega\right\} .
$$

Then $\left|\Omega^{\prime}\right|=1$. Now, for $u \in U_{p}$ satisfying

$$
-\Delta_{p} u=\tilde{\lambda}|u|^{p-2} u,
$$

define $v(y)=a^{1 / p} u\left(a^{1 / N} y\right)=a^{1 / p} u(x)$, with $y \in \Omega^{\prime}, x=a^{1 / N} y \in \Omega$. Then $\int_{\Omega^{\prime}}|v(y)|^{p}=1$, and $\nabla_{y} v(y)=a^{1 / N+1 / p} \nabla_{x} u(x)$. Therefore we have $-\Delta_{p} v=$ $\left(a^{p / N} \tilde{\lambda}\right)|v|^{p-2} v$, i.e. the new eigenvalue problem on the domain $\Omega^{\prime}$ is modified by a factor $a^{p / N}$, which is continuous in $p$.

\section{Continuity of $\lambda_{k}(p), k=1,2$}

We start with some preparation. Define the following sets:

$$
\begin{aligned}
A_{k}(p)=\{ & \sum_{i=1}^{k} \alpha_{i} \bar{u}(i, p): \sum_{i=1}^{k} \alpha_{i}=1, \alpha_{i} \geq 0, \\
& \bar{u}(i, p) \in\{u(i, p),-u(i, p)\}, i=1, \cdots, k\},
\end{aligned}
$$

and for $q \in(1, \infty)$,

$$
A_{k}^{q}(p)=\left\{u /\|u\|_{q} \in W_{0}^{1, q}(\Omega): u \in A_{k}(p)\right\} .
$$

First we have

Lemma 2.1. If $0 \notin A_{k}(p)$, then $A_{k}^{q}(p)$ is well defined and $\gamma\left(A_{k}^{q}(p)\right)=k$.

Proof. The regularity theory guarantees that for $u \in A_{k}(p), u \in W_{0}^{1, q}(\Omega)$. Observe that if $0 \notin A_{k}(p)$, then $A_{k}^{q}(p)$ is well defined and is symmetric and compact. We define a map $f_{k}: A_{k}^{q}(p) \rightarrow \boldsymbol{R}^{k} \backslash\{0\}$ as follows. Let $e_{i}^{k}, i=1, \cdots, k$, be the standard unit basis of $R^{k}$, define

$$
f_{k}\left( \pm u(i, p) /\|u(i, p)\|_{q}\right)= \pm e_{i}^{k},
$$

and for $u=\sum_{i=1}^{k} \alpha_{i} \bar{u}(i, p) /\left\|\sum_{i=1}^{k} \alpha_{i} \bar{u}(i, p)\right\|_{q}$,

$$
f_{k}(u)=\sum_{i=1}^{k} \alpha_{i} f_{k}\left(\bar{u}(i, p) /\|\bar{u}(i, p)\|_{q}\right) .
$$

$f_{k}$ so defined is an odd homeomorphism between $A_{k}^{q}(p)$ and the unit sphere of $\boldsymbol{R}^{k}$. It follows from Proposition 2.3 (a) of [Sz] that $\gamma\left(A_{k}^{q}(p)\right)=k$.

Corollary 2.1. If the eigenfunctions $u(i, p)$ are linearly independent, then $\gamma\left(A_{k}^{q}(p)\right)$ $=k$ for $k=1,2, \cdots$. 
In particular, since $u(1, p)$ is of one sign and $u(2, p)$ changes sign, obviously $0 \notin A_{1}(p) \cup A_{2}(p)$. Thus we have

Corollary 2.2. $\gamma\left(A_{k}^{q}(p)\right)=k$ for $k=1,2$.

From now on we consider the case $k=1,2$.

Lemma 2.2. $\lambda_{k}(p)$ is a bounded function of $p$ on any bounded interval $(1, s]$ for $k=1,2$.

Proof. By Corollary 2.2, $\gamma\left(A_{k}^{p}(s)\right)=k$. Observe that

$$
\lambda_{k}(p) \leq \sup _{v \in A_{k}^{p}(s)} \int|\nabla v|^{p} \leq \sup _{u \in A_{k}(s)} \frac{\int|\nabla u|^{p}}{\|u\|_{p}^{p}} \leq \sup _{u \in A_{k}(s)} \frac{\left(\int|\nabla u|^{s}\right)^{p / s}}{\|u\|_{p}^{p}} .
$$

Here we have used the assumption that $|\Omega|=1$. Note that $\inf _{u \in A_{k}(s)} \int|u|^{p}=$ $c(p, s)>0$. Indeed, if $\inf _{u \in A_{k}(s)} \int|u|^{p}=c(p, s)=0$, since $\int|\cdot|^{p}$ is a continuous function of $u$, and $A_{k}(s)$ is compact in $W_{0}^{1, s}(\Omega)$ and hence is compact in $W_{0}^{1, p}(\Omega)$, there exists a $u_{0} \in A_{k}(s)$ with $\int\left|u_{0}\right|^{p}=0$. This implies that $u_{0}=0$, a contradiction. Consequently we have

$$
0<\lambda_{k}(p) \leq \frac{\sup _{u \in A_{k}(s)}\left(\int|\nabla u|^{s}\right)^{p / s}}{\inf _{u \in A_{k}(s)} \int|u|^{p}} \leq\left[\lambda_{k}(s)\right]^{p / s} / c(p, s)<\infty .
$$

The lemma is proved.

Lemma 2.3. For $p, q \in(1, s], \lim _{p \rightarrow q} \inf _{u \in A_{k}(p)} \int|u|^{p}=1$.

Proof. Let $u(p) \in A_{k}(p)$ be such that

$$
u(p) \rightarrow \lim _{p \rightarrow q} \inf _{u \in A_{k}(p)} \int|u|^{p} .
$$

For $\varepsilon>0$ small, we have $p>q-\varepsilon$. Noting that $\int|\nabla u|^{q-\varepsilon} \leq\left(\int|\nabla u|^{p}\right)^{(q-\varepsilon) / p}$, Lemma 2.2 and the fact that $u(p)$ is a convex combination of $u(1, p)$ and $u(2, p)$ imply that $u(p)$ is bounded in $W_{0}^{1, q-\varepsilon}(\Omega)$. Consequently for a subsequence $\left\{p_{n}\right\}$ and some $u_{0} \in W_{0}^{1, q-\varepsilon}(\Omega), u\left(p_{n}\right) \rightarrow u_{0}$ weakly in $W_{0}^{1, q-\varepsilon}(\Omega)$ and strongly in $L^{q-\varepsilon}(\Omega)$. It then follows that

$$
\int\left|u_{0}\right|^{q-\varepsilon}=\lim _{p_{n} \rightarrow q} \int\left|u\left(p_{n}\right)\right|^{q-\varepsilon} \leq \lim _{p_{n} \rightarrow q}\left(\int\left|u\left(p_{n}\right)\right|^{p_{n}}\right)^{(q-\varepsilon) / p_{n}}=1 .
$$

Letting $\varepsilon \rightarrow 0$ we obtain $\int\left|u_{0}\right|^{q} \leq 1$.

On the other hand, Lemma 4.2 of [L2] implies that

$$
\int\left|u_{0}\right|^{p_{n}} \geq \int\left|u\left(p_{n}\right)\right|^{p_{n}}+p_{n} \int\left|u\left(p_{n}\right)\right|^{p_{n}-2} u\left(p_{n}\right)\left(u_{0}-u\left(p_{n}\right)\right) .
$$

The second integral on the right-hand side approaches 0 as $p_{n} \rightarrow q$. Thus $\int\left|u_{0}\right|^{q} \geq$ 1. The lemma is proved.

Now we are ready to prove our main result.

Theorem 2.1. $\lambda_{k}(p)$ is continuous in $p$ for $k=1,2$.

Proof. By Lemma 2.2, for any sequence $p_{n} \rightarrow q$, there exists a subsequence, still denoted by $\left\{p_{n}\right\}$, such that $\lambda_{k}\left(p_{n}\right) \rightarrow \tilde{\lambda}$. Our objective is to prove $\tilde{\lambda}=\lambda_{k}(q)$.

As in the proof of Theorem 4.1 of [L4], a standard diagonal argument produces a subsequence of $\left\{p_{n}\right\}$, still denoted by $\left\{p_{n}\right\}$, such that for some $u_{0} \in C^{1}(\Omega)$, 
$u\left(k, p_{n}\right) \rightarrow u_{0}$ and $\nabla u\left(k, p_{n}\right) \rightarrow \nabla u_{0}$ locally uniformly in $\Omega$. The boundedness of $\left\{\lambda_{k}\left(p_{n}\right)\right\}$ further implies that $u\left(k, p_{n}\right) \rightarrow u_{0}$ in $W_{0}^{1, q-\varepsilon}(\Omega)$ weakly for $\varepsilon>0$ small enough (cf. [L2], [L4]). Observe that

$$
\int\left|\nabla u\left(k, p_{n}\right)\right|^{p_{n}-2} \nabla u\left(k, p_{n}\right) \nabla \varphi=\lambda_{k}\left(p_{n}\right) \int\left|u\left(k, p_{n}\right)\right|^{p_{n}-2} u\left(k, p_{n}\right) \varphi
$$

for $\varphi \in C_{0}^{\infty}(\Omega)$. Letting $p_{n} \rightarrow q$ in (2.3), and noting that we have uniform convergence of $u\left(k, p_{n}\right)$ to $u_{0}$ on the support of $\varphi$, we obtain

$$
\int\left|\nabla u_{0}\right|^{q-2} \nabla u_{0} \nabla \varphi=\tilde{\lambda} \int\left|u_{0}\right|^{q-2} u_{0} \varphi
$$

whence we have $-\Delta_{q} u_{0}=\tilde{\lambda}\left|u_{0}\right|^{q-2} u_{0}$ and $u_{0} \in C^{1+\alpha}(\bar{\Omega})$. Moreover, the proof of Lemma 2.3 implies that $\left\|u_{0}\right\|_{q}=1$.

Observe that

$$
\begin{aligned}
\lambda_{k}(q) & \leq \sup _{v \in A_{k}^{q}\left(p_{n}\right)} \int|\nabla v|^{q}=\sup _{u \in A_{k}\left(p_{n}\right)} \frac{\int|\nabla u|^{q}}{\|u\|_{q}^{q}} \\
& \leq \frac{\sup _{u \in A_{k}\left(p_{n}\right)} \int|\nabla u|^{q}}{\inf _{u \in A_{k}\left(p_{n}\right)}\|u\|_{q}^{q}} .
\end{aligned}
$$

By (1.4) and the convergence of $u\left(k, p_{n}\right)$ to $u_{0}$, we know that for $p_{n}$ close enough to $q$ and $k=1,2, u\left(k, p_{n}\right) \in C^{1+\alpha}(\bar{\Omega})$ for some $\alpha>0$. Thus for $u \in A_{k}\left(p_{n}\right)$, $u \in C^{1+\alpha}(\bar{\Omega})$. Consequently for $\varepsilon>0$ such that $p_{n}+\varepsilon>q$, we have

$$
\int|\nabla u|^{q} \leq\left(\int|\nabla u|^{p_{n}+\varepsilon}\right)^{q /\left(p_{n}+\varepsilon\right)} \leq\left(\|\nabla u\|_{\infty}^{\varepsilon} \cdot \int|\nabla u|^{p_{n}}\right)^{q /\left(p_{n}+\varepsilon\right)}
$$

for $u \in A_{k}\left(p_{n}\right)$, and

$$
\left(\int|\nabla u|^{p_{n}}\right)^{1 / p_{n}} \leq \sum_{i=1}^{k} \alpha_{i}\left(\int|\nabla \bar{u}(i, p)|^{p_{n}}\right)^{1 / p_{n}} \leq \lambda_{k}(p) .
$$

Combining (2.5), (2.6) and (2.7), letting $p_{n} \rightarrow q$, then $\varepsilon \rightarrow 0$, and using Lemma 2.3, we conclude that

$$
\lambda_{k}(q) \leq \tilde{\lambda}
$$

On the other hand, we have

$$
\lambda_{k}\left(p_{n}\right) \leq \sup _{v \in A_{k}^{p_{n}}(q)} \int|\nabla v|^{p_{n}} .
$$

Hence the similar procedure yields

$$
\lambda_{k}(q) \geq \tilde{\lambda}
$$

Thus $\lambda_{k}(p)$ is continuous. This concludes the proof.

Remark 2.1. The continuity of $\lambda_{1}(p)$ is proved by Lindqvist in [L4]. For the O.D.E. case, the explicit formula of $\lambda_{k}(p)$ is given in [GV]; see also [DEM] and [HM].

Remark 2.2. Our proof breaks down for the case $k \geq 3$ because it is not clear for this case whether $0 \in A_{k}(p)$ or not. However, our proof shows that, if all the (variational) eigenfunctions are linearly independent, then $\lambda_{k}(p)$ is continuous with respect to $p$, since then $0 \notin A_{k}(p)$ for all $k>0$. We note that the independence of the eigenfunctions is a standing open question for the $p$-Laplacian for $p \neq 2$. 


\section{Asymptotic Behavior of $\lambda_{1}(p)$}

We discuss the asymptotic behavior of $\lambda_{1}(p)$ as $p \rightarrow 1$ and $p \rightarrow \infty$.

Theorem 3.1. Assume $B\left(x_{0}, \tau\right) \subset \Omega$. Then

$$
\lambda_{1}(p) \leq \frac{(N+p) \cdots(p+1)}{N ! \tau^{p}} .
$$

Moreover, $\lim _{p \rightarrow \infty} \lambda_{1}(p)=0$ if $\tau>1$.

Proof. Take $\varphi=\tau-\left|x-x_{0}\right|$ if $\left|x-x_{0}\right| \leq \tau$, and $\varphi=0$ if $\left|x-x_{0}\right|>\tau$. Then

$$
\lambda_{1}(p) \leq \frac{\int|\nabla \varphi|^{p}}{\int|\varphi|^{p}} .
$$

Calculation shows that

$$
\int|\nabla \varphi|^{p}=\frac{\tau^{N}}{N} \frac{2 \pi^{N / 2}}{\Gamma(N / 2)}
$$

and

$$
\int|\varphi|^{p}=\frac{2 \pi^{N / 2}}{\Gamma(N / 2)} \tau^{p+N} \int_{0}^{1}(1-s)^{p} s^{N-1} d s,
$$

where $\Gamma(\cdot)$ is the $\Gamma$-function. It is known that

$$
\int_{0}^{1}(1-s)^{p} s^{N-1} d s=\frac{\Gamma(N) \Gamma(p+1)}{\Gamma(N+p+1)}=\frac{(N-1) !}{(N+p) \cdots(p+1)},
$$

hence we obtain

$$
\lambda_{1}(p) \leq \frac{(N+p) \cdots(p+1)}{N ! \tau^{p}} .
$$

The theorem then follows.

We will see later that the condition $\tau>1$ is optimal.

To obtain an estimate for $\lambda_{1}(p)$ as $p \rightarrow 1$, we first establish the following Barta type inequality.

Lemma 3.1. For any positve $v \in C_{0}^{2}(\Omega)$, let $\beta=\inf _{x \in \Omega}\left(-\Delta_{p} v / v^{p-1}\right)$. Then $\lambda_{1}(p) \geq \beta$. Moreover $\lambda_{1}(p)>\beta$ if $v \neq c u(1, p)$, where $c$ is a constant.

Proof. We write $u_{1}=u(1, p)$ for short. Observe that

$$
-\Delta_{p} u_{1}=\lambda_{1}(p) u_{1}^{p-1},
$$

and

$$
-\Delta_{p} v \geq \beta v^{p-1}
$$

By using the monotonicity of $-\Delta_{p} \cdot$ and $(\cdot)^{p-1}$, and integrating by parts, we obtain

$$
\begin{aligned}
0 & \leq \int\left(\left|\nabla u_{1}\right|^{p-2} \nabla u_{1}-|\nabla v|^{p-2} \nabla v\right)\left(\nabla u_{1}-\nabla v\right) \\
& \leq\left(\lambda_{1}(p)-\beta\right) \int\left(u_{1}^{p-1}-v^{p-1}\right)\left(u_{1}-v\right) .
\end{aligned}
$$

This implies the desired inequality. The proof is completed. 
Theorem 3.2. Assume that $|\Omega|=\delta$. Then for $1<p<2$,

$$
\lambda_{1}(p) \geq \frac{(N+p-2)}{(2(p-1) / p)^{p-1}}\left(\frac{p}{2-p}\right)^{1-p / 2} \tau^{-p-1},
$$

where $\tau=\left(2 \pi^{N / 2}\right)^{-1 / N}[N \Gamma(N / 2) \delta]^{1 / N}$. Moreover, $\lim _{p \rightarrow 1} \lambda_{1}(p) \geq(N-1) \tau^{-2}$.

Proof. Let $\Omega^{*}$ be the symmetrization of $\Omega$, i.e. $\Omega^{*}$ is radially symmetric and $\left|\Omega^{*}\right|=$ $\delta$. Then $\Omega^{*}=B(0, \tau)$. Let $\lambda_{1}^{*}(p)>0$ be the first eigenvalue of the $p$-Laplacian on $\Omega^{*}$; then $\lambda_{1}^{*}(p) \leq \lambda_{1}(p)$ (cf. Corollary 2.33 of [K2]). Next we estimate $\lambda_{1}^{*}(p)$.

Take $v=\tau^{2}-|x|^{2}$ in $\Omega^{*}$. Then $-\Delta_{p} v=2^{p-1}(N+p-2)|x|^{p-2}$. Let $\beta=$ $\inf \left(-\Delta_{p} v / v^{p-1}\right)$. One easily checkes that, for $1<p<2, \beta$ is attained at $|x|=$ $((2-p) / p)^{1 / 2} \tau$ and

$$
\beta=\frac{(N+p-2)}{(2(p-1) / p)^{p-1}}\left(\frac{p}{2-p}\right)^{1-p / 2} \tau^{-p-1} .
$$

The theorem then follows from the above estimate and Lemma 3.1.

We now turn our attention to the one dimensional case and obtain more accurate asymptotic behavior. It is known (see, e.g., Theorem 1 of $[\mathrm{GV}]$, and [DEM]) that the $k$-th eigenvalue of the problem

$$
-\left(\left|u^{\prime}\right|^{p-2} u^{\prime}\right)^{\prime}=\lambda(p)|u|^{p-2} u, \quad u(0)=u(L)=0
$$

is given by

$$
\begin{aligned}
\lambda_{k}(p) & =k^{p} \lambda_{1}(p)=k^{p} L^{-p}(p-1)\left[2 \int_{0}^{1}\left(1-t^{p}\right)^{-1 / p} d t\right]^{p} \\
& =k^{p}(p-1)\left[\frac{2}{L} \cdot \frac{\pi}{p \sin (\pi / p)}\right]^{p} .
\end{aligned}
$$

Theorem 3.3. (i) $\lim _{p \rightarrow \infty} \lambda_{1}(p)=\infty$ if $L \leq 2$. (ii) $\lim _{p \rightarrow \infty} \lambda_{1}(p)=0$ if $L>2$. (iii) $\lim _{p \rightarrow 1^{+}} \lambda_{1}(p)=2 / L$.

Proof. For $L \neq 2$, (i) and (ii) follow from the fact that $\lim _{p \rightarrow \infty} \pi(p \sin (\pi / p))^{-1}=1$.

For $L=2$, we observe that, since $\ln (\pi / p)-\ln (\sin (\pi / p))>0$,

$$
\ln \left[(p-1)^{1 / p} \frac{\pi}{p \sin (\pi / p)}\right]^{p}=p\left[\ln \frac{\pi / p}{\sin (\pi / p)}+\frac{1}{p} \ln (p-1)\right] \rightarrow+\infty
$$

as $p \rightarrow \infty$. So $\lambda_{1}(p) \rightarrow \infty$.

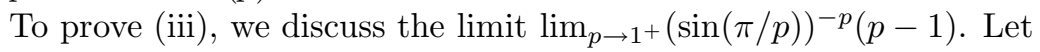

$$
y=(\sin (\pi / p))^{p}
$$

then

$$
\begin{aligned}
\ln y & =p \ln \sin (\pi / p) \\
& =p \ln \left[\frac{\sin (\pi / p)}{(p-1) \pi}\right]+p \ln [(p-1) \pi]=o(1)+p \ln [(p-1) \pi] .
\end{aligned}
$$

Therefore $y=e^{o(1)}((p-1) \pi)^{p}$. Observe that

$$
\begin{aligned}
\lim _{p \rightarrow 1^{+}} \frac{p-1}{(\sin (\pi / p))^{p}} & =\lim _{p \rightarrow 1^{+}} \frac{(p-1)}{e^{o(1)}(p-1)^{p} \pi^{p}} \\
& =\lim _{p \rightarrow 1^{+}} \frac{1}{e^{o(1)} \pi^{p}} \frac{1}{(p-1)^{p-1}}=\frac{1}{\pi} .
\end{aligned}
$$

(iii) then follows from (3.4). The theorem is proved. 
Remark 3.1. We see from (i) that the condition $\tau>1$ in Theorem 3.1 cannot be improved in general. Using (3.4), we can also obtain asymptotic behavior for other $\lambda_{k}(p)$.

\section{REFERENCES}

[AA] J.P. G. Azorero and I.P. Alonso, Existence and uniqueness for the p-Laplacian: nonlinear eigenvalues, Comm. PDE 12 (1987), 1389-1430.

[DEM] M. Del Pino, M. Elgueta and R. Manasevich, A homotopic deformation along $p$ of a LeraySchauder degree result and existence for $\left(\left|u^{\prime}\right|^{p-2} u^{\prime}\right)^{\prime}+f(t, u)=0, u(0)=u(T)=0, p>1$, J. Diff. Equa. 80 (1989), 1-13. MR 91i:34018

[D] J.I. Diaz, Nonlinear Partial Differential Equations and Free Boundaries vol 1: Elliptic Equations, Research Notes in Mathematics 106, Pitman Advanced Publishing Program, London, 1985. MR 88d:35058

[Eg] H. Egnell, Existence and nonexistence results for $m$-Laplace equations involving critical Sobolev exponents, Arch. Rat. Mech. Anal. 104 (1988), 57-77. MR 90e:35069

[GT] D. Gilbarg and N.S. Trudinger, Elliptic Partial Differential Equations of Second Order, 2nd edition, Springer-Verlag, N.Y., 1983. MR 86c:35035

[GV] M. Guedda and L. Veron, Bifurcation phenomena associated to the p-Laplace operator, Trans. Amer. Math. Soc. 310 (1988), 419-431. MR 89j:35024

[HM] Y.X. Huang and G. Metzen, The existence of solutions to a class of semilinear differential equations, Diff. Int. Equa. 8 (1995), 429-452. MR 95h:34034

[K1] B. Kawohl, On a family of torsional creep problems, J. Reine Angew. Math. 410 (1990), 1-22. MR 91h:35126

[K2] B. Kawohl, Rearrangements and Convexity of Level Sets in PDE, Lecture Notes in Math. \# 1150, Springer-Verlag, N.Y. 1985. MR 87a:35001

[LM] A.C. Lazer and P.J. McKenna, Large-amplitude periodic oscillations in suspension bridges: some new connections with nonlinear analysis, SIAM Review 32 (1990), 537-578. MR 92g:73059

[L1] P. Lindqvist, Stability for the solutions of div $\left(|\nabla u|^{p-2} \nabla u\right)=f$ with varying $p$, J. Math. Anal. Appl. 127 (1987), 93-102. MR 88i:35057

[L2] P. Lindqvist, On the equation div $\left(|\nabla u|^{p-2} \nabla u\right)+\lambda|u|^{p-2} u=0$, Proc. Amer. Math. Soc. 109 (1990), 157-164. MR 90h:35088

[L3] P. Lindqvist, Note on a nonlinear eigenvalue problem, Helsinki University of Technology, Inst. of Math. Research Reports \# A277; Rocky Mountain J. Math. 23 (1993), 281-288. MR 94d:34031

[L4] P. Lindqvist, On a nonlinear eigenvalue problem: stability and concavity, Helsinki University of Technology, Inst. of Math. Research Reports \# A279.

[R] P.H. Rabinowitz, Minimax Methods in Critical Point Theory with Applications to Differential Equations, AMS Regional Conference Series in Math. vol. 65 (1986). MR 87j:58024

[Sz] A. Szulkin, Ljusternik-Schnirelmann theory on $C^{1}$-manifolds, Ann. Inst. Henri Poincaré, Anal. Nonl. 5 (1988), 119-139. MR 90a:58027

[T] P. Tolksdorf, On the Dirichlet problem for quasilinear equations in domains with conical boundary points, Comm. PDE 8 (1983), 773-817.

Department of Mathematical Sciences, University of Memphis, Memphis, Tennessee 38152

E-mail address: huangy@mathsci.msci.memphis.edu 